\title{
Adiponectin and Its Role in Inflammatory Process of Obesity
}

\author{
Ami Febriza ${ }^{1,2}$, Ridwan $^{3}$, Suryani As'ad ${ }^{4}$, Vivien Novarina Kasim ${ }^{5}$ Hasta Handayani Idrus ${ }^{6}$ \\ ${ }^{1}$ Department of Physiology, Faculty of Medicine, Universitas Muhammadiyah Makassar, Makassar, Indonesia \\ ${ }^{2}$ Postgraduate Program of Medical Science, Faculty of Medicine, Universitas Hasanuddin, Makassar, Indonesia \\ ${ }^{3}$ Mappa Oudang Nursing Academy, Makassar, Indonesia \\ ${ }^{4}$ Department of Clinical Nutrition, Faculty of Medicine, Universitas Hasanuddin, Makassar, Indonesia \\ ${ }^{5}$ Faculty of Sport and Health, Universitas Negeri Gorontalo, Gorontalo, Indonesia \\ ${ }^{6}$ Department of Microbiology, Faculty of Medicine, Universitas Muslim Indonesia, Makassar, Indonesia
}

Obesity is a chronic, low degree systemic inflammatory status. Microarray examination shows a disturbance in the expression of cytokine, chemokine, complementary protein and half of the other acute phase components in obese patients. Adiponectin is the hormone that increases insulin sensitivity, while its level decreases under condition of fatty tissue enlargement that occurs in obesity. Excessive weight causes the adipocyte cells and adipose tissues produce various types of mediators. The inflammatory process is the main cause of metabolic diseases, and the main role of adipose tissue in the inflammatory process is determined by the production of pro-inflammatory mediators and anti-inflammatory mediators. Adiponectin has an important anti-inflammatory effect on obesity. Adiponectin has an important anti-inflammatory effect on obesity. Adiponectin works on macrophage and monocyte to inhibit the production of pro-inflammatory cytokine and increase the expression of interleukin (IL)-10 and IL-1 receptor antagonists. Adiponectin reduces induction of intercellular adhesion molecule (ICAM)-1 and vascular cell adhesion molecule (VCAM)-1 endothelial adhesion by TNF- $\alpha$ or resistin. In obese patients, it is characterized by resistance to adiponectin alongside a decrease and the possibility of adiponectin loss in the receptor population in liver and muscles, leading to low adiponectin level.

Keywords: adiponectin, obesity, inflammation

\section{Introduction}

Overweight and obese are disorder or disease characterized by excessive accumulation of fat tissue in the body. ${ }^{1}$ Overweight is defined as having a body mass index (BMI) between 25 and 29.9, while obese is defined as having a BMI of 30 or higher. Overweight and obese people, who are characterized by fat deposits, have experienced a chronic low-grade systemic inflammatory process. The results of microarray examination in obese patients indicate a disturbance in the expression of cytokine, chemokine, complementary protein and half of the other acute phase components. It has also been suggested that the theory that activation of non-specific immune system components

Date of submission: February 8, 2019

Last Revised: March 7, 2019

Accepted for publication: March 8, 2019

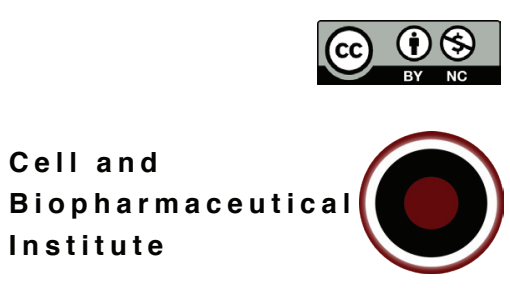

Corresponding Author:

Ami Febriza

Department of Physiology, Faculty of Medicine

Universitas Muhammadiyah Makassar

Gunung Sari, Rappocini, Makassar 90221, Indonesia

E-mail: amifebriza@med.unismuh.ac.id 
can be considered a factor that allows the development of obesity and inflammation associated with it. These mediators provide important systemic effects may lead to insulin resistance, metabolic disorder and cardiovascular disease in obese patients. ${ }^{2}$

Obesity pathomechanism is based on the theory of inflammation, which is characterized by lipid accumulation in adipose tissue and expansion of fat mass leading to the initiation of the inflammatory process. It starts with adipose cells producing pro-inflammatory cytokines and chemokine, including tumor necrosis factor (TNF)- $\alpha$, interleukin (IL)-6, leptin, resistin, monocyte chemoattractant protein (MCP)1 , and plasminogen activator inhibitor (PAI)-1. Vascular endothelial cells respond through increased expression of adhesion molecules, which together with chemokine will attract immune cells, including monocyte-macrophages towards adipose tissue. Together, adipose cells, immune cells and endothelial cells and the resultant mediators will create an inflammatory environment that triggers insulin resistance. Pro-inflammatory mediators and proatherogenic mediators then enter the circulation, causing insulin resistance and increasing the risk of atherosclerosis. ${ }^{3}$

Adiponectin, an adipocytokine, has an important anti-inflammatory effect on obesity. In obese patients, it is characterized by resistance to adiponectin along with a decrease and the possibility of loss of adiponectin receptor population in liver and muscles, leading to low level of adiponectin. Adiponectine resistance occurs due to downregulation of adiponectin receptors (AdipoRs). Low level of adiponectin as anti-inflammatory will tend to aggravate the inflammatory process in obese people. Another report states that adiponectin is a key element at the molecular level that mediates the occurrence of insulin resistance in obese population. ${ }^{4}$

The study was conducted by Eizadi, et al., in obese adult men, with an analysis using the Independent $\mathrm{T}$ test. $^{5}$ The samples show lower adiponectin level in obese men compared to men with normal weight $(5.44 \pm 1.23$ in the obese group and 7.56 \pm 1.32 in the normal weight group, $p=0.021$ ). Based on research by Bacha, et al., in obese adults, there is a decrease in adiponectin level at around 50\% compared to adults of normal weight. ${ }^{6}$ In the obese adult group with more visceral fat tissues, they tend to have lower adiponectin level compared to obese people with less visceral fat tissues (6.2 \pm 0.9 and $9.0 \pm 1.0 \mu \mathrm{g} / \mathrm{mL}, p=0.05)$. This review intends to provide an explanation of the relationship and mechanism of adiponectin in the inflammatory process in obesity.

\section{Adiponectin Molecules}

Adiponectin is one of the adipocytokines that was first discovered in 1995 by Scherer. Adiponectin is also known as adipocyte complement related protein 30 kilodalton (ACRP 30), AdipoQ, adipose most abundant gene transcript (apM)-I and gelatin binding protein 28 kilodalton (GBP 28). ${ }^{7}$ Adiponectin has gene mapping on chromosome $3 \mathrm{q} 27$. Several analyzes of single nucleotide polymorphisms (SNPs) and missense mutations, it was found that adiponectin gene is associated with the metabolic syndrome. ${ }^{8}$

\section{Adiponectin Structure}

Adiponectin is a protein formed from 247 amino acids consisting of 4 parts, namely amino acid terminals, variable regions, collagenous domains (cAd) and globular carboxy/ globular terminal C-terminal domains of adiponectin (gAd). Adiponectin includes a collagen superfamily that dissolves and has a homologous structure with collagen VIII and X factor, Clq complement factor and TNF family. ${ }^{9}$

$\mathrm{X}$-ray crystallography from gAd reveals a homologous structure with TNF- $\alpha$. This indicates a link between evolutionary development between TNF- $\alpha$ and adiponectin. Both of these components have the opposite function, namely TNF- $\alpha$ as proinflammatory and adiponectin as antiinflammatory. ${ }^{7}$

The basic form of adiponectin is a trimer which is formed by three rnonomer bonds in the globular domain. The monomer structure is not found to be circulated but is retained in adipocytes. Four to six trimers form a higher structure called oligomers with plasma concentrations of $5-30 \mu \mathrm{g} / \mathrm{L}{ }^{7}$

Plasma level of adiponectin ranges from 3.0-30 $\mu \mathrm{g} / \mathrm{L}$, whereas adiponectin levels in cerebrospinal liquor is reported in $1-4 \%$ of serum level. Although it is not yet clear whether adiponectin can pass through the blood-brain barrier, there is evidence that mammalian adiponectin can. The half-life of adiponectin is approximately 14 hours. ${ }^{10}$

Yamauchi separates 2 adiponectin receptors, AdipoRl and AdipoR2. Adipo R1 is produced more in skeletal muscles and adipoR2 in the liver. AdipoRl mediates the activation of peroxisome proliferators activator receptor (PPAR)- $\alpha$, adenosin monophosphate activated proteinkinase (AMPK), glucose and $\beta$ oxidation uptake, thus increasing gluconeogenesis. AdipoR2 is involved in the activation of PPAR nuclear receptors to mediate $\beta$ oxidation and reception of reactive oxygen species (ROS).? 
Two forms of receptors have been cloned for adiponectin with distinct distribution and affinity for the molecular form of protein. AdipoR1 is a receptor with high affinity for gAd, in contrast with full-length adiponectin (fAd), whilst AdipoR2 has intermedia affinity for both forms of adiponectin. AdipoR1 is expressed in skeletal muscle, while AdipoR2 is mainly expressed throughout the liver. Such finding is in line with observations that suggest that fAd has a greater effect on metabolic signals in the liver. Adiponectin may have an anti-tumor effect by acting as a negative regulator of angiogenesis by inducing endothelial cell apoptosis mediated by caspase. This effect is seen not to be affected by AMP-kinase intermediate signals. Besides, adiponectin has anti-inflammatory, anti-fibrotic effects on liver tissue, anti-diabetic and anti-lipidemic. AdipoR2 was found first in the liver, where AdipoR1 was reported to be found previously in skeletal muscles. Adiponectin level in plasma is significantly lower in obesity. ${ }^{11}$

\section{Adiponectin Secretion}

Adiponectin is synthesized in white fat cell tissues (adipocytes) and is produced during fat cell differentiation. The results show that adipose tissues are not only a place for storing fat, but also are endocrine organs that play an important role in interaction with endocrine, metabolic and inflammatory signals to regulate the body's energy homeostasis. ${ }^{12}$ Adiponectin was first discovered while examining gene expression in human's visceral and subcutaneous fat tissue, which was aimed to determine the mechanism of diseases associated with obesity. Unexpectedly, genes expressed in subcutaneous and visceral fat tissue, at $20 \%$ and $30 \%$, are genes that produce various kinds of bioactive secretory proteins (bioactive secretory protein), which are then called adipocytokine. One of these adipocytokines is adiponectin. . $^{13,14}$

\section{Factors affecting Adiponectin Level}

Gender influences the metabolic and endocrine function of adipose tissues. Women have a greater percentage of body fat than men. Increased adiposity in women is associated with larger fat cell size, increased lipolysis stimulation, increased triglyceride (TG) synthesis in this depot. This finding implies that female hormones play a significant role in these depot differences between genders, resulting in differences in adipocyte metabolism. Estrogen is a clear candidate and may mediate several differences in adipocyte metabolism. Sexual dimorphism is also related to differences in the distribution of the adiponectin complex in circulation. ${ }^{15,16}$

A cross-sectional study involving 705 men, 262 women in Japan, ages 30-65 years, BMI of $22.5 \pm 2.9 \mathrm{~kg} /$ $\mathrm{m}^{2}$. Serum adiponectin concentration was measured using enzyme-linked immunosorbent assay (ELISA) method. Serum adiponectin concentration obtained in women $(13.5 \pm 7.9 \mu \mathrm{g} / \mathrm{L})$ was significantly higher than men $(7.2 \pm 4.6$ $\mu \mathrm{g} / \mathrm{L}) .{ }^{17}$ Adiponectin level is higher in women. It is assumed that influence of sexual hormone regulates adiponectin production. $^{18}$

Factors that also affect plasma adiponectin level are including circadian rhythm, showing the regulation of diurnal and pulsatile adiponectin secretion in humans. Adiponectin level reaches their peak in the morning and declines at night. ${ }^{19}$ Other factors that affect the expression and secretion of adiponectin are dietary factors. Fish oil and linoleic acid are also able to increase plasma adiponectin level, which is in line with the fact that consumption of these substances will provide a protective effect on diabetes mellitus. ${ }^{20}$ In contrast, diets with high carbohydrates and fats can reduce plasma adiponectin level. ${ }^{21}$ In addition, level of adiponectin plasma is negatively correlated with BMI, insulin resistance, TG, and low density lipoprotein (LDL) and is positively correlated with high density lipoprotein (HDL). ${ }^{17}$ High level of plasma adiponectin concentration were also found in thin individuals and patients with type 1 diabetes mellitus, and level is low in patients with type 2 diabetes mellitus and obese individuals. ${ }^{22}$

\section{Adiponectin as Anti Inflammation}

The role of adiponectin and TNF- $\alpha$ inhibits the production of one another in adipose tissues. C-reactive protein (CRP) expression is negatively regulated by adiponectin in fat tissues. Adiponectin expression is suppressed by IL-6 in fat tissues. Adiponectin inhibits monocyte adhesion and TNF$\alpha$-induced adhesion molecular expression, macrophage transformation into foam cells, TNF- $\alpha$ expression in macrophage and smooth muscle cell proliferation. ${ }^{12,23}$

Adiponectin can improve the negative impact of TNF- $\alpha$ on endothelial function. Without the need to inhibit the bonds of TNF- $\alpha$ and fAd, it can inhibit the adhesion molecules induced by TNF- $\alpha$, VCAM-I, E-selectin and ICAM-I. Adiponectin suppresses inflammatory changes by inhibiting inhibitory nuclear factor kappa B (NF-kB) phosphorylation and NF-kB activation without affecting 
activation of c-Jun N-terminal kinase (JNK), p38 and Akt which is activated by TNF- $\alpha$. Adiponectin inhibits leukocyte colony formation, thus decreasing phagocytic activity and TNF- $\alpha$ secretion. ${ }^{24}$

A Study reported that adiponectin inhibits cell proliferation induced by oxidized-LDL (oxLDL), inhibits superoxide secretion-induced, and activation of p42/p44 MAPK by oxLDL. The impact of circulating oxLDL on the vascular wall results in foam cell formation, inactivation of endothelial nitric oxide (eNo), induction of inflammatory responses and formation of ROS. All these components are known to play an active role in the atherogenesis process. ${ }^{24}$

\section{The Inflammatory Process induced by Changes in Adipose Tissues in Obesity}

Obesity conditions are associated with chronic inflammation characterized by response to abnormal cytokine production and activation of inflammatory signaling pathways. Adipose tissues in obese patients are characterized by progressive inflammation and infiltration by macrophages. Changes in the size of adipocyte and fat cells cause physical changes in the surrounding area and modification of the paracrine function of adipocytes. In obesity, adipocyte cells will release low level of TNF- $\alpha$, which can stimulate preadipocyte cells to produce chemoattractant, namely MCP-1. Similarly, endothelial cells also secrete MCP-1 in response to cytokines. Therefore, both preadipocytes and endothelial cells can be responsible for attracting macrophages to adipose tissues. ${ }^{25}$

Adiposopathy is the accumulation of ectopic pathogenic adipocytes due to positive energy, sedentary, genetic, and environmental balance. The manifestation is a combination of adipocytes which are hypertrophy and ectopic, especially in the visceral. Such combination causes immunological and metabolic disorders. ${ }^{26}$ Adipocytes have dramatically varying cell sizes. Cell diameter can be about ten times as large and its volume becomes a thousand times bigger. This size affects the level of inflammation, lipid mobilization, and the pattern of adipocyte secretion. The spread of adipocytes in the abdomen is called the Android obesity pattern and those around the hips and thighs are called gynoid obesity pattern. From several studies, it is known that the form of android often describes fat accumulation in visceral organs associated with metabolic disorders including dyslipidemia, hypertension, and glucose intolerance. ${ }^{27}$

Adipose tissues in non-obese individuals consist of a number of inflammatory cells and secretes various active substances; however, adipose tissues in obese individuals show more accumulation of macrophages and $\mathrm{T}$ cells, resulting in excessive amount of inflammatory mediators, such as MCP-1 and IL-6, and low adiponectin secretion. Increasingly large adipocyte cells will trigger stress in the endoplasmic reticulum (ER). Such condition is important in triggering inflammatory kinases, such as JNK and I-kappa-B kinase (IKK), which ultimately inhibit insulin signals and activate inflammatory cascades and inflammatory mediator production. The available evidence demonstrates that increased chemokine production, such as MCP-1 in obesity adipose tissues can increase the accumulation of macrophages. In tissues, monocytes and macrophages can be sources that secrete TNF- $\alpha$. Cytokines, such as TNF- $\alpha$ and other stimuli, can lead to further activation of inflammatory kinases. Several studies have shown that $\mathrm{T}$ cells also accumulate in adipose tissues in obese individuals. Interferon-gamma (IFN $\gamma$ ), a typical T-helper 1 cytokine which possibly regulates the expression of TNF- $\alpha$, MCP-1, and other inflammatory mediators, shows a role for adaptive immunity in the pathophysiology of obesity. Adipokines, such as IL-6, into the circulation can also trigger important systemic effects, such as increased production of acute inflammatory mediator in the liver and coagulation factors, which are likely to correlate with the incidence of atherothrombosis. ${ }^{2}$

Increased secretion of leptin (and/or decreased production of adiponectin) secreted by adipocyte cells can also cause accumulation of macrophages by stimulating transport of macrophages towards adipose tissues and will also increase adhesion of macrophages to vascular endothelial cells. Physical damage to the endothelium, caused both by size changes and oxidative damage due to the more lipolytic environment, plays a role in the recruitment and accumulation of macrophage, similar to those seen in atherosclerosis. Any initial stimulus attracts macrophages into adipose tissue, but once these macrophage cells have infiltrated adipose tissue and become active, the macrophages together with adipocyte cells and other cell types will form a vicious circle of accumulation of larger withdrawal of macrophages, inflammatory production cytokines, and cause impaired adipocyte cell function ${ }^{25}$

Adipogenesis is the process by which precursor cells (preadipocytes) diffuse into mature adipocytes. During diffusion, there are morphological changes, cell development, fat accumulation, changes in insulin sensitivity, and adipokine expression. ${ }^{28}$ This mass 
enlargement of adipocyte is a compensation for the insulin defect. Each adipocyte mass has a specific expression of the gene and responds differently to food, hormones, and temperature. Metabolically, active adipocytes found in the intra-abdominal, intra-muscular, perivascular and epicardial serve to provide energy for vital organs, such as liver, heart, blood vessels, and muscular muscles. However, the adipocytes in the omentum serve as sensors to regulate the diets eaten and distribute them from liver to the body through the mediation of autonomic neurons. ${ }^{29}$

Apart from its anatomical location, the main difference between visceral and subcutaneous adipocytes is found in anabolic response, efficiency, proliferation, and in vitro differentiation. In mammals, adipocytes consist of 2 types, which are white adipose tissue (WAT) and brown adipose tissue (BAT). WAT and BAT are divided on the basis of metabolic characteristics. WAT mainly serves to store excess energy for subsequent needs, whilst BAT is an energy-wasting organ. In experimental mice, BAT plays an important role to prevent and reduce obesity through increased energy disposal and heat production. The role of BAT in humans is unclear, because the number has drastically reduced in newborns; whereas in adults, 1 BAT is compared to $100-200$ WAT. $^{30}$

There are two forms of macrophages in adipocytes, i.e., type M1 macrophage which serves to remove TNF- $\alpha$, IL6, and increase inflammation; whilst M2-type macrophage serves to release anti-inflammatory cytokine, such as IL10 and repair tissues. Both of these macrophages decrease alongside the decrease in body weight. ${ }^{31}$ In some situations, macrophages can increase up to $40 \%$ in adipocytes. The mechanism of macrophage mobilization is unclear, possibly involving chemotaxic molecular secretion, and one of them is the $\mathrm{C}-\mathrm{C}$ motif chemokine ligand 2 (CCL2) known as MCP1. CCL2 is the main C-C ligand of the chemokine receptor 2 (CCR2) motive. ${ }^{32}$ As a result of adipocyte enlargement, the blood flow decreases; and if it cannot be compensated for by angiogenesis, there will be a disruption of perfusion and triglyceride clusters, followed by hypoxia. Hypoxia will stimulate the expression of angiogenic transcription factors, decreasing the expression of adiponectin, and PPAR- $\partial$. Then adipocyte releases low level of TNF- $\alpha$ and stimulates preadipocyte to produce MCP-1. MCP-1 secretion aims to respond to the secretion of cytokine, leptin, and/ or decreased adiponectin secretion. All of these conditions can cause accumulation and increase in adhesion of macrophages to endothelial cells and adiposity. ${ }^{25}$
TNF- $\alpha$ is the most important inflammatory mediator secreted by macrophages. TNF- $\alpha$ enhances the induction of other proinflammatory molecules, such as IL-6, IL8, MCP-1, and IL-1. ${ }^{33}$ Obstructing the development and differentiation of preadipocytes can be done by interfering with the insulin signal through a signaling mechanism of tyrosine phosphorylation, inhibiting serine phosphorylation, and down-regulation of several insulin signaling pathways. All of these conditions might result in insulin resistance, increase lipolysis, and decrease glucose uptake by adipocytes. The addition of anti TNF- $\alpha$ can inhibit inflammation in preadipocyte; and in adipocytes, dietary regulation in experimental animals can reduce MCP-1 and macrophages. $^{33,34}$

The biggest abnormality in visceral free fatty acid metabolism is the inability to suppress lipolysis due to hyperinsulinemia. ${ }^{35}$ Free fatty acids in the tissues will stimulate the production of destructive metabolite and cause structural abnormalities, necrosis, systemic inflammation, and endoplasmic reticulum stress. The saturated fatty acid bond with the 4 th-like receptor-like macrophage (TLR-4) will activate NF-kB and TNF- $\alpha{ }^{36}$ Besides stimulating $\beta$-cell apoptosis, albumin increases expression of uncoupling protein (UCP)-2 and can further inhibit the production of adenosine triphosphate (ATP) needed for insulin secretion. ${ }^{37}$

\section{The Relationship between Adiponectin and Inflammatory Process in Obesity}

Previous research. revealed two important findings: first, obesity and type 2 diabetes were associated with low plasma adiponectin concentration and showed that this hypoadiponectinemia was evident in various ethnic groups with clear differences with the tendency in the obese group, type diabetes 2, and atherosclerosis. Second, the result shows that plasma adiponectin concentration is more closely related to insulin sensitivity and insulinemia level in fasting individuals compared with adiposity and glycemic condition. It suggests that hypoadiponectinemia in people with obesity and type 2 diabetes is largely due to insulin resistance and/ or hyperinsulinemia. ${ }^{22}$

The results of other studies in Japanese individuals have shown that plasma adiponectin concentrations are negatively correlated with BMI and therefore the level is lower in obese subjects than thin subjects. ${ }^{38-40}$ These results indicate that plasma adiponectin concentration is inversely related to the percentage of body fat and similar condition is also found in various ethnic groups. Adiponectin is one 
of the protein secreted by adipose. Despite being produced exclusively in white adipose tissue, adiponectin leves is low in obesity. This condition is also consistent with findings in mice, murine homologue of adiponectin, adipoQ which also experience down-regulation in obesity. ${ }^{41}$

\section{Conclusion}

Adiponectin is the hormone that increases insulin sensitivity and decreases levels in condition of fatty tissue enlargement occured in obesity. Adiponectin has an important antiinflammatory effect on obesity. Adiponectin works on macrophage and monocyte to inhibit the production of pro-inflammatory cytokine and increase the expression of IL-10 and IL-1 receptor antagonists. Adiponectin reduces induction of ICAM-1 and VCAM-1 endothelial adhesion by TNF- $\alpha$ or resistin. In obese patients, it is characterized by resistance to adiponectin together with a decrease and possible loss of adiponectin receptor population in the liver and muscles, leading to low adiponectin level. Low level of adiponectin as anti-inflammatory will tend to aggravate the inflammatory process in obese people. In contrast to adiponectin, leptin is a hormone released by adipocyte cells. If the amount of adipose tissue increases, adipocyte cells will produce more leptin; therefore the leptin concentration in plasma is positively associated with the mass of fatty tissues.

\section{Acknowledgements}

I am deeply indebted to my supervisor, Prof. dr. Suryani As'ad, M.Sc, Sp.GK(K), senior lecturer from Department of Clinical Nutrition, Universitas Hasanuddin, for a warm support, inspiration and thoughtful guidance in writing this article. I would like to thank to Universitas Muhammadiyah Makassar for giving such an enthusiasm and support in this process of writing.

\section{References}

1. Yussac MAA, Cahyadi A, Putri AC, Dewi AS, Khomaini A, Bardosono S, et al. Prevalensi obesitas pada anak usia 4-6 tahun dan hubungannya dengan asupan serta pola makan. Maj Kedokt Indones. 2007; 57(2): 47-53

2. Rocha VZ, Folco EJ. Inflammatory concepts of obesity. Int J Inflam. 2011; 2011: 529061. doi: 10.4061/2011/529061.

3. Shoelson SE, Herrero L, Naaz A. Obesity, inflammation, and insulin resistance. Gastroenterology. 2007; 132(6): 2169-80.

4. Kadowaki T, Hara K, Yamauchi T, Terauchi Y, Tobe K, Nagai R. Molecular mechanism of insulin resistance and obesity. Exp Biol Med. 2003; 228(10): 1111-7.
5. Eizadi M, Khorshidi D, Doali H. Relationship between serum adiponectin with anthropometrical and lipid profile biochemical indexes in obese adult men. Int Conf Environ Biomed Biotechnol. 2011; 16: 70-3.

6. Fida Bacha M, Rola Saad M, Neslihan Gungor M, Silva A, Arslanian M. Adiponectin in youth: relationship to visceral adiposity, insulin sensitivity, and b-cell function. Diabetes Care. 2004; 27(2): 547-52.

7. Kadowaki T, Yamauchi T. Adiponectin and adiponectin receptors. Endocr Rev. 2009; 26(3): 439-51.

8. Okamoto Y, Kihara S, Funahashi T, Matsuzawa Y, Libby P. Adiponectin: a key adipocytokine in metabolic syndrome. Clin Sci. 2006; 110(3): 267-78.

9. Kadowaki T, Yamauchi T, Kubota N, Hara K, Ueki K, Tobe K. Adiponectin and adiponectin receptors in insulin resistance, diabetes, and the metabolic syndrome. J Clin Invest. 2006; 116(7): 1784-92.

10. Peterlin BL, Bigal ME, Tepper SJ, Urakaze M, Sheftell FD, Rapoport AM. Migraine and adiponectin: is there a connection? Cephalalgia. 2007; 27(5): 435-46.

11. Manju Chandran M, Susan A, Phillips M, Theodore Ciaraldi P, Robert R, Henry M. Adiponectin: more than just another fat cell hormone? Diabetes Care. 2003; 26(8): 2442-50

12. Matsuzawa Y, Funahashi T, Kihara S, Shimomura I. Adiponectin and metabolic syndrome. Arterioscler Thromb Vasc Biol. 2004; 24(1): 29-33.

13. Kern PA, Di Gregorio GB, Lu T, Rassouli N, Ranganathan G. Adiponectin expression from human adipose tissue: relation to obesity, insulin resistance, and tumor necrosis factor-alpha expression. Diabetes. 2003; 52(7): 1779-85.

14. Ntambi JM, Kim Y. Adipocyte differentiation and gene expression. J Nutr. 2000; 130(12): 3122-6.

15. Al-hashem F, Ibrahim I, Bastawy N, Rateb M, Haidara M, Dallak M, et al. Effect of insulin on adiponectin and adiponectin receptor-1 expression in rats with streptozotocin-induced type 2 diabetes. $\mathrm{J}$ Health Sci. 2011; 57(4): 334-40.

16. Ronti $T$, Lupattelli $G$, Mannarino $E$. The endocrine function of adipose tissue: an update. Clin Endocrinol. 2006; 64(4): 355-65.

17. Yamamoto Y, Hirose H, Saito I, Tomita M. Correlation of the adipocyte-derived protein adiponectin with insulin resistance index and independent of body mass index , in the Japanese population. Clin Sci. 2002; 103(2): 137-42.

18. Xu A, Chan KW, Hoo RLC, Wang Y, Tan KCB, Zhang J, et al. Testosterone selectively reduces the high molecular weight form of adiponectin by inhibiting its secretion from adipocytes. J Biol Chem. 2005; 280(18): 18073-80.

19. Gavrila A, Peng C, Chan JL, Mietus JE, Goldberger ARYL, Mantzoros $\mathrm{CS}$, et al. Diurnal and ultradian dynamics of serum adiponectin in healthy men: comparison with leptin, circulating soluble leptin receptor, and cortisol patterns. J Clin Endocrinol Metab. 2003; 88(6): 2838-43

20. Nagao K, Inoue N, Wang YM, Yanagita T. Conjugated linoleic acid enhances plasma adiponectin level and alleviates hyperinsulinemia and hypertension in Zucker diabetic fatty (fa/fa) rats. Biochem Biophys Res Commun. 2003; 310(2): 562-6.

21. Pischon T, Girman CJ, Rifai N, Hotamisligil GS, Rimm EB. Association between dietary factors and plasma adiponectin concentrations in men 1 - 3. Am J Clin Nutr. 2005; 81 (4): 780-6.

22. Weyer C, Funahashi T, Tanaka S, Hotta K, Matsuzawa Y, Pratley RE, et al. Hypoadiponectinemia in obesity and type 2 diabetes: close 
association with insulin resistance and hyperinsulinemia. J Clin Endocrinol Metab. 2001; 86(5): 1930-5.

23. Ouchi N, Walsh K. A novel role for adiponectin in the regulation of inflammation. Arterioscler Thromb Vasc Biol. 2008; 28(7): 121921.

24. Goldstein BJ, Scalia R. Adiponectin: a novel adipokine linking adipocytes and vascular function. J Clin Endocrinol Metab. 2004; 89(6): 2563-8.

25. Wellen KE, Hotamisligil GS. Obesity-induced inflammatory changes in adipose tissue. J Clin Invest. 2003; 112(12): 1785-8.

26. Bays HE, González-Campoy JM, Henry RR, Bergman DA, Kitabchi $\mathrm{AE}$, Schorr $\mathrm{AB}$, et al. Is adiposopathy (sick fat) an endocrine disease? Int J Clin Pract. 2008; 62(10): 1474-83.

27. Kissebah AH, Krakower GR. Regional adiposity and morbidity. Physiol Rev. 1994; 74(4): 761-811.

28. Rosen ED, Hsu CH, Wang X, Sakai S, Freeman MW, Gonzalez FJ, et al. C/EBPalpha induces adipogenesis through PPARgamma: a unified pathway. Genes Dev. 2002; 16(1): 22-6.

29. Kreier F, Kap YS, Mettenleiter TC, van Heijningen C, van der Vliet J, Kalsbeek A, et al. Tracing from fat tissue, liver, and pancreas: a neuroanatomical framework for the role of the brain in type 2 diabetes. Endocrinology. 2006; 147(3): 1140-7.

30. Oberkofler H, Dallinger G, Liu YM, Hell E, Krempler F, Patsch W. Uncoupling protein gene: quantification of expression levels in adipose tissues of obese and non-obese humans. J Lipid Res. 1997; 38(10): 2125-33.

31. Lumeng CN, Bodzin JL, Saltiel AR. Obesity induces a phenotypic switch in adipose tissue macrophage polarization. J Clin Invest. 2007; 117(1): 175-84.

32. Charo IF, Taubman MB. Chemokines in the pathogenesis of vascular disease. Circ Res. 2004; 95(9): 858-66.

33. Lacasa D, Taleb S, Keophiphath M, Miranville A, Clement K.
Macrophage-secreted factors impair human adipogenesis: involvement of proinflammatory state in preadipocytes. Endocrinology. 2007; 148(2): 868-77.

34. Kanda H, Tateya S, Tamori Y, Kotani K, Hiasa K, Kitazawa R, et al. MCP-1 contributes to macrophage infiltration into adipose tissue, insulin resistance, and hepatic steatosis in obesity. J Clin Invest. 2006; 116(6): 1494-505.

35. Jensen MD, Caruso M, Heiling V, Miles JM. Insulin regulation of lipolysis in nondiabetic and IDDM subjects. Diabetes. 1989; 38(12): 1595-601.

36. Suganami T, Tanimoto-Koyama K, Nishida J, Itoh M, Yuan X, Mizuarai S, et al. Role of the Toll-like receptor 4/NF-kappaB pathway in saturated fatty acid-induced inflammatory changes in the interaction between adipocytes and macrophages. Arterioscler Thromb Vasc Biol. 2007; 27(1): 84-91.

37. Zhou H, Zhao J, Zhang X. Inhibition of uncoupling protein 2 by genipin reduces insulin-stimulated glucose uptake in 3T3-L1 adipocytes. Arch Biochem Biophys. 2009; 486(1): 88-93.

38. Ouchi N, Kihara S, Arita Y, Maeda K, Kuriyama H, Okamoto Y, et $a l$. Novel modulator for endothelial adhesion molecules: adipocytederived plasma protein adiponectin. Circulation. 1999; 100(25): 2473-6.

39. Arita Y, Kihara S, Ouchi N, Takahashi M, Maeda K, Miyagawa J, et al. Paradoxical decrease of an adipose-specific protein, adiponectin, in obesity. Biochem Biophys Res Commun. 1999; 257(1): 79-83.

40. Hotta K, Funahashi T, Arita Y, Takahashi M, Matsuda M, Okamoto $\mathrm{Y}$, et al. Plasma concentrations of a novel, adipose-specific protein, adiponectin, in type 2 diabetic patients. Arterioscler Thromb Vasc Biol. 2000; 20(6): 1595-9.

41. Hu E, Liang P, Spiegelman BM. AdipoQ is a novel adipose-specific gene dysregulated in obesity. J Biol Chem. 1996; 271(18): $10697-$ 703. 